\title{
Optimization of processing technology of Rhizoma Pinelliae Praeparatum and its anti-tumor effect
}

\author{
Xiuting Zhang, Yongjiang Cai, Lijing Wang, Hongchun Liu, Xiulin Wang
}

First affiliated hospital of Zhengzhou University Department of Medicine, Zhengzhou, Henan Province, China

\begin{abstract}
Background: Rhizoma Pinelliae is the dried tuber of Pinellia ternata (Thunb.) Breit. Modern pharmacological studies have shown that Rhizoma Pinelliae has antitussive, antiemetic, glandular secretion inhibiting and antitumor effects.

Objectives: To optimize the processing technology of Rhizoma Pinelliae Praeparatum, and to study its anti-tumor effect. Methods: Orthogonal design method was applied to analyze the effects of factors such as licorice concentration volume, soaking time and processing temperature on processing technology of Rhizoma Pinelliae Praeparatum; MTT assay and flow cytometry were used to determine the inhibitory effect of Rhizoma Pinelliae Praeparatum on Bel-7402 cells.

Results: During the processing of Rhizoma Pinelliae Praeparatum, the size of influence of licorice concentration volume, soaking time and processing temperature on processing results of Rhizoma Pinelliae was: $\mathrm{B}>\mathrm{C}>\mathrm{A}$ in descending order, i.e. soaking time $>$ processing temperature $>$ licorice concentration volume, different concentrations of Rhizoma Pinelliae Praeparatum ethanol extracts could all exert inhibitory effect on the growth and proliferation of Bel-7402 cells, and with the increase of drug concentration and the extension of culture time, the cell proliferation inhibitory effect of Rhizoma Pinelliae Praeparatum ethanol extract became more and more evident. Apoptotic rate of $1.5 \mathrm{mg} / \mathrm{ml}$ Rhizoma Pinelliae Praeparatum ethanol extract group reached $13.53 \%$, the difference was extremely significant compared with the control group. In conclusion the factor most influential to the processing technology of Rhizoma Pinelliae Praeparatum was soaking time, followed by processing temperature, the factor least influential was licorice concentration volume.
\end{abstract}

Conclusion: Rhizoma Pinelliae Praeparatum has inhibitory effect on growth and proliferation of Bel-7402 cells.

Keywords: Rhizoma Pinelliae Praeparatum, orthogonal experiment, Bel-7402 cells

DOI: http://dx.doi.org/10.4314/ahs.v15i1.14

\section{Introduction}

Rhizoma Pinelliae is the dried tuber of Pinellia ternata (Thunb.) Breit. in the family Araceae, which was originally recorded in the "Shen Nong's Herbal Classic" as a low-grade drug. ${ }^{1-2}$ It is acrid in taste, warm in nature, toxic, and enters the spleen, stomach and lung meridians. It has the effects of drying dampness and resolving phlegm, regulating middle warmer and invigorating stomach, descending adverse-rising qi and arresting vomiting, relieving stuffiness and dissipating nodulation. It is commonly used in the clinical treatment of stuffy chest, thoracic obstruction, chest bind, upward perversion of stomach qi, nausea, vomiting, excessive phlegm, cough, etc. ${ }^{2-5}$ Modern pharmacological studies

\section{Corresponding author: \\ Cai Yong \\ First affiliated hospital of Zhengzhou \\ University Department of Medicine, \\ Zhengzhou, Henan Province, China \\ E-Mail: cuviner@163.com}

have shown that Rhizoma Pinelliae has antitussive, antiemetic, glandular secretion inhibiting and antitumor effects. $^{6-8}$

The processing of Rhizoma Pinelliae has long been recorded by physicians of past dynasties, which was first seen in Zhang Zhongjing's "Jin Kui Yu Han Jing": "Wash in hot water for a dozen times until clear water is seen, toxic if not washed thoroughly." After the Song Dynasty, the processing technology of Rhizoma Pinelliae was further developed, and the types of adjuvant materials were further enriched. For example, "processing with alum" in the "Prescriptions of Peaceful Benevolent Dispensary" 'Processing with Ginger Juice and Alum', General Records", processing with licorice and ginger in "Zhu Shi", Ming Dynasty's processingwith licorice and lime in the "Compendium of Materia Medica", and processing with ethanol and ginger in the "Prescriptions for Universal Relief", Qing Dynasty's "Supplement to Compendium of Materia Medica" recorded a method of processing with lime, alum and saltpeter. 
Crude Rhizoma Pinelliae is strongly irritant, so in most cases Rhizoma Pinelliae is used in clinic as processed products. The fundamental purpose for processing traditional Chinese Medicine is to attenuate toxicity and enhance efficacy. The processing of Rhizoma Pinelliae Praeparatum is a typical representative. Through different approaches of processing, toxic constituents of Rhizoma Pinelliae are reduced, thus extending its range of clinical applications. There are 4 types of processed products included in the 2010 edition of "Chinese Pharmacopoeia", namely the unprocessed Rhizoma Pinelliae, Rhizoma Pinelliae Praeparatum, ginger-processed Rhizoma Pinelliae and alum-processed Rhizoma Pinelliae, of which Rhizoma Pinelliae Praeparatum is strong in eliminating cold phlegm, and is a commonly used Chinese medicine in clinic. In this paper, the factors influencing the processing technology of Rhizom Pinelliae Praeparatum were optimized, in the meanwhile, its anti-cancer activity was investigated.

\section{Methods}

\section{Drugs and reagents}

Rhizoma Pinelliae and Radix Glycyrrhizae were purchased from the market, which were identified as the dried tuber of Pinellia ternata (Thunb.) Breit. in the family Araceae by professor kanghui who from zhengzhou University; and the root and rhizome of Glycyrthiza uralensis Fisch. in the family Leguminosae. RPM 1640 (Gibco); RNase enzyme, PI (propidium iodide) cator (Sigma), MTT (Sigma, USA).

Table 1: Factors and levels of orthogonal experimen

\begin{tabular}{cccc}
\hline Level & $\begin{array}{c}\text { A Licorice } \\
\text { concentration volume } \\
(\mathrm{mL})\end{array}$ & B Soaking time (d) & $\begin{array}{c}\text { C Processing } \\
\text { temperature }\left({ }^{\circ} \mathrm{C}\right)\end{array}$ \\
\hline \hline 1 & 100 & 5 & 25 \\
2 & 200 & 6 & 30 \\
3 & 300 & 7 & 40 \\
\hline
\end{tabular}

Experiment on inhibition of Bel-7402 cell proliferation by Rhizoma Pinelliae Praeparatum ethanol extract

Preparation of Rhizoma Pinelliae Praeparatum ethanol extract

Rhizoma Pinelliae Praeparatum was taken, ground,

and ultrasonically extracted twice with 59\% ethanol,

each extraction lasted $2 \mathrm{~h}$, the extracts were combined, Cell culture medium was RPMI 1640 medium contain-

Human hepatoma cell line Bel-7402 was purchased from Shanghai Ruiqi Biological Technology Co., Ltd.

\section{Methods}

Processing preparation of Rhizoma Pinelliae

Raw Rhizoma Pinelliae of relatively uniform size were taken, and soaked in adequate amount of water until

\section{Factors and levels of orthogonal experiment}

During the processing of Rhizoma Pinelliae, licorice concentration volume, soaking time and processing temperature had relatively great influences on the processing results and efficacy of Rhizoma Pinelliae. In ducting experiment in accordance with the L9(3)3 factor-level table, and with the content of mono-ammonium glycyrrhizinate in each processed product determined by ultraviolet spectrophotometry and high performance liquid chromatography as the indi-

rough micropous and the remaining was filtere with RPMI 1640 medium to make into $0.5,1$ and 1.5 $\mathrm{mg} / \mathrm{ml}$ Rhizoma Pinelliae Praeparatum ethanol extract ng $10 \%$ fetal calf serum, $100 \mathrm{U} / \mathrm{mL}$ penicillin and 100 Effect of Rhizoma Pinelliae Praeparatum ethano $\mu \mathrm{g} / \mathrm{mL}$ streptomycin. The cells were cultured under the conditions of $37^{\circ} \mathrm{C}, 5 \% \mathrm{CO} 2$ and saturated humidity, and digested and passaged with $0.25 \%$ trypsin, cells after three passages were used in the experiment. there was no dry core inside, then removed, and dried in the air. Experiment was performed in accordance with

Determination of inhibition rate of Rhizom Pinelliae Praeparatum ethanol extract on Bel-7402 cell proliferation by MTT assay

Bel-7402 cells in the logarithmic growth phase were taken, digested with trypsin for $2 \mathrm{~min}$ and collected, the collected Bel-7402 cells were then seeded in 96-well plates at a concentration of $5 \times 104$ cells $/ \mathrm{mL}, 5$ replicate wells were set up for each group, each well was with $100 \mu \mathrm{L}$ of cell solution, after culturing for $12 \mathrm{~h}$, medium was changed, the experimental groups were added with $0.5,1$ and $1.5 \mathrm{mg} / \mathrm{ml}$ ethanol extracts of Rhizoma Pinelliae Praeparatum, while the blank control group was added only with an equal volume of culture medium. After incubating under the conditions of $37^{\circ} \mathrm{C}$, $5 \% \mathrm{CO} 2$ and saturated humidity for $24 \mathrm{~h}$, each well was added with $25 \mu \mathrm{L}$ ofMTT $(5 \mathrm{mg} / \mathrm{mL}$ ), and the incubation was continued for another $4 \mathrm{~h}$, then supernatan was discarded, $150 \mu \mathrm{L}$ of DMSO was added, followed by shaking. Optical density (OD) value was measured at $490 \mathrm{~nm}$ wavelength, and cell growth inhibition rate was calculated.

Inhibition rate $\%)=(\mathrm{OD}$ value of the negative control roup - OD value of the treatment group)/(OD value of the negative control group - OD value of the blank control group)

Table 2: Orthogonal experimental results

\begin{tabular}{ccccc}
\hline No. & A & B & C & $\begin{array}{c}\text { Mono-ammonium } \\
\text { glycyrrhizinate yield (\%) }\end{array}$ \\
\hline 1 & 1 & 1 & 1 & 0.13 \\
2 & 1 & 2 & 2 & 0.17 \\
3 & 1 & 3 & 3 & 0.36 \\
4 & 2 & 1 & 2 & 0.12 \\
5 & 2 & 2 & 3 & 0.21 \\
6 & 2 & 3 & 1 & 0.16 \\
7 & 3 & 1 & 3 & 0.13 \\
8 & 3 & 2 & 1 & 0.16 \\
9 & 3 & 3 & 2 & 0.27 \\
\hline k1 & 0.66 & 0.38 & 0.45 & \\
k2 & 0.49 & 0.54 & 0.56 & \\
k3 & 0.56 & 0.79 & 0.70 & \\
\hline K1 & 0.22 & 0.12 & 0.15 & \\
K2 & 0.16 & 0.18 & 0.19 & \\
K3 & 0.19 & 0.26 & 0.23 & \\
R & 0.06 & 0.14 & 0.08 & \\
\hline
\end{tabular}

The logarithmic growth phase Bel-7402 cells were taken, and cultured for $24 \mathrm{~h}$, then different concentration of Effect of Rhizoma Pinelliae Praeparatum ethanol extracts were added, respectively, and the culti-

vation was continued for another $12 \mathrm{~h}$. The cells were then digested with $0.25 \%$ trypsin, washed with PBS, centrifuged to remove supernatant, and fixed with $70 \%$ ce-cold ethanol. Before detection, the cells were centrifuged, washed with PBS, followed by discarding of sernatant. The cells were stained with PI, kept in dark place at room temperature for $20 \mathrm{~min}$, then detected by flow cytometry, analyzed using CELLQuest software,

\section{Results}

Optimization of processing technology of Rhizoma Pinelliae Praeparatum

Orthogonal experimental results showed that during the processing of Rhizoma Pinelliae Praeparatum, the size of influence of licorice concentration volume, solking time and processing temperature on processing esults of Rhizoma Pinelliae was: $\mathrm{B}>\mathrm{C}>\mathrm{A}$ in descending order, i.e. soaking time $>$ processing temperature $>$ liane analysis, the optimal technology for processing of Rhizoma Pinelliae Praeparatum was determined as:

B 3 C3, i.e. licorice concentration volume of $100 \mathrm{ml}$ soaking duration of 7 days, and processing temperature of $40^{\circ} \mathrm{c}$. 
Inhibitory effect of Rhizoma Pinelliae Praepara- Praeparatum ethanol extracts could all exert inhibitory tum ethanol extract on proliferation of Bel-7402 effect on the growth and proliferation of Bel-7402 cells,

As can be seen by the MTT assay results, different and with the increase of drug concentration and the extension of culture time, the cell proliferation inhibitory effect of Rhizoma Pinelliae Praeparatum ethano extract became more and more evident. (See Fig. 1)

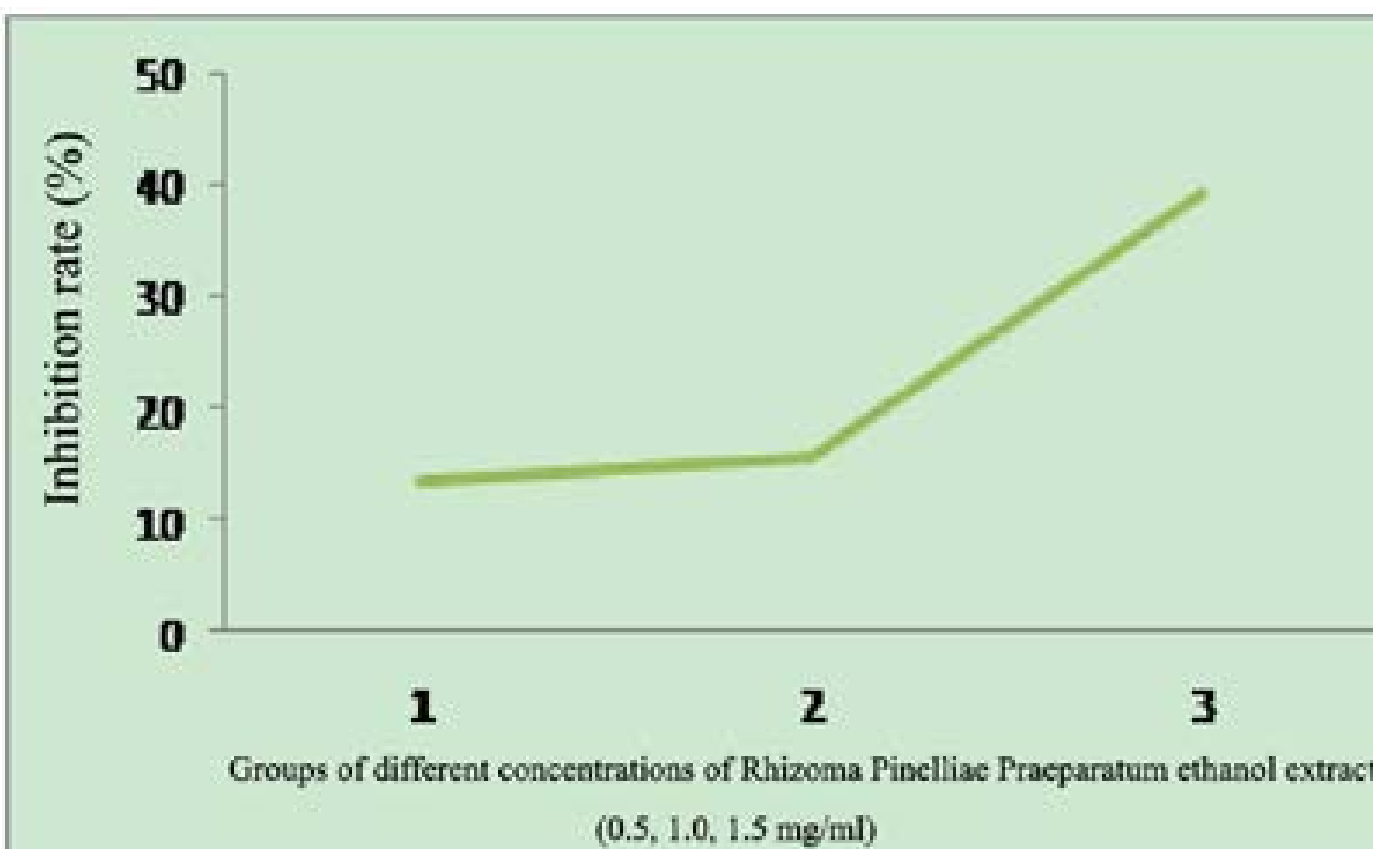

Figure 1: Inhibitory effect of Rhizoma Pinelliae Praeparatum ethanol extract on Bel-7402

cell proliferation

Induction of Bel-7402 cell apoptosis by Rhizoma Pinelliae Praeparatum ethanol extract

showed a significant dose-effect relationship. Apoptotic rate of $1.5 \mathrm{mg} / \mathrm{ml}$ Rhizoma Pinelliae Praeparatum ethAs can be seen from Tab. 3, different concentrations of anol extract group reached $13.53 \%$, the difference was Rhizoma Pinelliae Praeparatum ethanol extracts could extremely significant compared with the control group. all effectively induce apoptosis of Bel-7402 cells, which

Table 3: Effect of Rhizoma Pinelliae Praeparatum ethanol extract on apoptosis of Bel-7402 cells $(\overline{\mathrm{x}} \pm \mathrm{s})$

\begin{tabular}{cccc}
\hline Group & Concentration $(\mathrm{mg} / \mathrm{ml})$ & $\mathrm{n}$ & Apoptotic $(\%)$ \\
\hline Control group & & 6 & $3.45 \pm 1.78$ \\
Rhizoma Pinelliae & 0.5 & 6 & $6.57 \pm 1.52^{*}$ \\
Praeparatum ethanol & 1 & 6 & $7.89 \pm 1.97^{*}$ \\
extract group & 1.5 & 6 & $13.53 \pm 1.16^{* *}$ \\
\hline
\end{tabular}

Note: Comparison with the control group, ${ }^{*} \mathrm{P}<0.05, * * \mathrm{P}<0.01$
The process of apoptosis can be divided into three Malignant tumor is the chief culprit causing harm to phases morphologically: the first is the phase of apopthe health of patients today. According to statistics, tosis initiation: manifestations are disappearance of miabout 2.2 million people contract various types of can- crovilli and other specialized structures of cell surface, cer every year worldwide, and about 0.8 million of them but cell membrane is still intact, without losing selective receive chemotherapy, while treating cancer, also greatly permeability; in the cytoplasm, mitochondria are basi-

harming the patients' body, the search of effective an- cally intact, endoplasmic reticulum lumen is expanded, ti-cancer divg and methods is an inportant task faced by global medical field. The medical field found during the search and use of anti-cancer drugs that many chemical anti-cancer drugs tend to affect normal cells while acting on target cells, in addition, most chemical drugs used for clinical treatment of cancer have different degrees of mutagenic genetic toxicity. Natural med icine is characterized by multi-component, multi-target, multi-function and low toxicity, therefore, the search of safe and effective anticancer drugs with small toxic and side effects from natural substances has become focus of researches on anticancer drugs of scholars at home and abroad ${ }^{9-1}$

The toxic constituents of unprocessed Rhizoma Pinelliae are yet unclear. But it has been confirmed that its toxicity is mainly manifested in strong irritating effect on oral and gastrointestinal mucosa, which can cause palpebral conjunctival edema in rabbits, thereby causing gastric mucosal injury. In this study, the factors influenceng the processing technology of Rhizoma Pinelliae Praeparatum, one of the processed products of Rhizoma Pinelliae, were optimized. As can be seen by the analysis of orthogonal experimental results, after investigating three main factors influencing the processing of Rhizoma Pinelliae Praeparatum, namely the licorice concentration volume, soaking time and processing temperature, it was found that the size of influence on processing results of Rhizoma Pinelliae was: $\mathrm{B}>\mathrm{C}>\mathrm{A}$ in descending order, i.e. soaking time $>$ processing temperature $>$ licorice concentration volume, based on the results and range analysis, the optimal technology for processing of Rhizoma Pinelliae Praeparatum was determined as: $\mathrm{A} 1 \mathrm{~B} 3 \mathrm{C} 3$, i.e. licorice concentration volume of $100 \mathrm{ml}$, soaking duration of 7 days, and processing temperature of $40^{\circ} \mathrm{c}$.

There exists balance between cell proliferation and death in the normal body, the two are not only mutually contradictory, but also maintain body homeostasi together, cell proliferation, differentiation and apoptosis are regulated jointly, in order to maintain normal growth of tissue. and gradually integrated with the plasma membrane; chromatin is pyknotic, forming a crescent shape, and is distributed along the nuclear membrane. The second is the phase of apoptotic body formation: nuclear chromatin is cleaved into fragments of varying sizes, aggregates with organelle, and is gradually separated, forming individual apoptotic bodies. The third is the phase of apoptotic body phagocytosis: the apoptotic bodies are gradually engulfed and digested by phagocytic cells such as macrophages and epithelial cells. ${ }^{11-12}$

As can be seen by the MTT assay results, different concentrations of Rhizoma Pinelliae Praeparatum ethanol extracts could all exert inhibitory effect on the growth and proliferation of Bel-7402 cells, and with the increase of drug concentration and the extension of culture time, the cell proliferation inhibitory effect of Rhizoma Pinelliae Praeparatum ethanol extract became increasingly evident. In addition, Rhizoma Pinelliae Praeparatum ethanol extracts could all effectively induce apoptosis of Bel-7402 cells, which showed a significant dose-effect relationship.

Apoptotic rate of $1.5 \mathrm{mg} / \mathrm{ml}$ Rhizoma Pinelliae Praeparatum ethanol extract group reached $13.53 \%$, the difference was extremely significant compared with the control group. The experimental results demonstrate that ethanol extract of Rhizoma Pinelliae Praeparatum can effectively inhibit the proliferation of Bel-7402 cells, and induce their apoptosis, ultimately achieving the killing effect on human hepatoma Bel-7402 cells.

\section{References}

1. Hu SL. A survey on the medicinal history of Pinellia ternata Breit. China Journal of Chinese Materia Medica. 1989; 14(11): 646-348.

2. Wang J. Study on the toxicity of Rhizoma Pinelliae and attenuation by processing. Shaanxi Journal of Traditional Chinese Medicine. 2010; 31(9): 1229-1229.

3. Xiu YF, Wang ZH, Hong XK. Research Progress on Toxicity of Rhizoma Pinelliae. Lishizhen Medicine and Materia Medica Research. 2004; 15(5): 304-306. 4. Wang $\mathrm{CK}, \mathrm{Mu}$ WJ. Experimental investigation on processing of Rhizoma Pinelliae. Journal of Henan College 
of Traditional Chinese Medicine. 2001; 16(4): 12-13.

5. Wu H. Evolution of Rhizoma Pinelliae Processing from Han to Song Dynasties. Journal of Nanjing University of Traditional Cbinese Medicine. 2001; 17(1): 45-45.

6. Zheng GC. A study on the anti-tumor activity of Rhizoma Pinelliae extract. Journal of Sichuan Traditional Chinese Medicine. 2004; 22(9): 9-11.

7. Gui L, Gui SQ, Chen SH, Ge XR, Lin LG, Ye Y. Apoptosis induction effect of Pinellia extract fraction on cell lines of cervical cancer cultured in vitro. China Journal of Traditional Chinese Medicine and Pharmacy. 2008; 23(5): 447-449.

8. Wang GF. Effect of Pinellia pedatisecta Schott effective extract on in vitro cultured HepG2 cells. Chinese Journal of Modern Drug Application. 2009; 3(12): 113-114.
9. Haeker G. The morphology of aPoPtosis. Cell Tissue Res,. 2000; 301(1): 5-17.

10. Joza N, Susin SA, Daugas E, Stanford WL, Cho SK, Li CY, Sasaki T, Elia AJ, Cheng HY, Ravagnan L, Ferri KF, Zamzami N, Wakeham A, Hakem R, Yoshida H, Kong YY, Mak TW, ZunigaPflucker JC, Kroemer G, Penninger JM. Essential role of the mitochondrial apoptosis-inducing factor in programmed cell death. Nature. 2001; 410: 549-554.

11. Du Y, Wang XL, Wu GX, Wang YJ, Yang HC, Zuo LF. Flow cytometric detection and significance of four cyclins in esophageal cancer. Chinese Journal of Oncology, 2004; 26(10): 612-614.

12. Sato T, Friend C, De Harven E. Ultrastructural changes in Friend erythroleukemia cells treated with dimethyl sulfoxide. Cancer Res. 1971; 31(10): 1402-1417. 